\title{
New Exact Non-relativistic Energy Eigen Values for Modified Inversely Quadratic Hellmann Plus Inversely Quadratic Potential
}

\section{Maireche A*}

Department of Physics, Laboratory of Physics and Material Chemistry, University of M'sila, M'sila, Algeria

\begin{abstract}
In this current research, the solutions of modified Schrödinger equation (MSE) are presented for two companied potentials namely: modified inversely quadratic Hellmann potential and modified inversely quadratic potential (MIHQP), using generalization of Bopp's shift method (instead to solving MSE with star product) and standard perturbation theory in extended quantum mechanics (EQM), we obtained modified Hamiltonian operator and corresponding modified eigenvalues in both three dimensional noncommutative space and phase (NC-3D: RSP) symmetries.
\end{abstract}

Keywords: Inversely quadratic hellmann; Inversely quadratic potential; Non-commutative space and phase; Star product

\section{Introduction}

It is well known that, the ordinary Schrodinger equation is one of the fundamental wave equations in physics. Recently, considerable efforts have been made towards obtaining exact analytic solution of MSE for central potentials in two and three dimensional space in different fields of nuclear physics, spectroscopy, quantum chemistry and many fields of matter sciences to search an profound physical and chemical interpretations at Nano and Plank's scales [1-9], and in particularly our works in this context [10-29]. The algebraic physical structure of EQM based on the following fundamental four NC canonical commutations relations (NCCRs), in both Schrödinger and Heisenberg pictures (SP and HP), respectively, as ( $c=\hbar=1)$ [1-12],

$$
\begin{aligned}
& {\left[\hat{x}_{i}^{*}, \hat{p}_{j}\right]=i \delta_{j},\left[\hat{x}_{i}^{*}, \hat{x}_{j}\right]=i \theta_{j} \text { and }\left[\hat{p}_{i}^{*}, \hat{p}_{j}\right]=i \bar{\theta}_{j}} \\
& {\left[\hat{x}_{i}(t)^{*}, \hat{p}_{j}(t)\right]=i \delta_{j},\left[\hat{x}_{i}(t)^{*}, \hat{x}_{j}(t)\right]=i \theta_{j} \text { and }\left[\hat{p}_{i}(t)^{*}, \hat{p}_{j}(t)\right]=i \bar{\theta}_{j}}
\end{aligned}
$$

The very small two parameters $\theta^{\mu v}$ and $(*)$ (compared to the energy) are elements of two antisymmetric real matrixes and $(*)$ denote to the new star product, which is generalized between two arbitrary functions $g(x, p)$ and $g(x, p)$ to $(f * g)(x, p)$ instead of the old product $(f g)(x, p)$ [6-15]

$$
\delta(f * g)(x, p)=-\frac{i}{2}\left\{\theta^{\mu v} \partial_{\mu}^{x} f \partial_{\nu}^{x} g+\bar{\theta}^{\mu v} \partial_{p}^{x} f \partial_{p}^{x} g\right\}(x, p)
$$

Where $\delta(f * g)(x, p) \equiv(f * g-f g)(x, p)$, the new canonical coordinates $\hat{x}_{i}(t)$ and new momentum $\hat{p}_{i}(t)$ are determined from two projection relations, respectively, as follows [16],

$$
\left(\hat{x}_{i}(t), \hat{p}_{i}(t)\right)=\exp \left(i H_{n c}\left(t-t_{0}\right)\right) *\left(\hat{x}_{i}, \hat{p}_{i}\right) * \exp \left(-i H_{n c}\left(t-t_{0}\right)\right)
$$

By differentiating equation (3), we find that general two operators $\left(\hat{x}_{i}(t), \hat{p}_{i}(t)\right)$ obey the Heisenberg equations of motion [16]:

$$
\frac{d \hat{x}_{i}(t)}{d t}=i\left[H_{n c}{ }^{*}, \hat{x}_{i}(t)\right] \text { and } \frac{d \hat{p}_{i}(t)}{d t}=i\left[H_{n c}{ }^{*}, \hat{p}_{i}(t)\right]
$$

The formalism of star product, Bopp's shift method and the Seiberg-Witten map were played crucial roles in EQM. The Bopp's shift method will be apply in this paper instead of solving MSE in global group symmetry (GGS) (NC-3D:RSP), the MSE will be treated by using directly the two new commutators, in addition to usual ordinary commutators on quantum mechanics, in the both SP and HP representation, respectively [17-22],

$$
\left[\hat{x}_{i}, \hat{x}_{j}\right]=\left[\hat{x}_{i}(t), \hat{x}_{j}(t)\right]=i \theta_{i j} \text { and }\left[\hat{p}_{i}, \hat{p}_{j}\right]=\left[\hat{p}_{i}(t), \hat{p}_{j}(t)\right]=i \bar{\theta}_{i j}
$$

the new two operators $p_{i}$ and $p_{i}$ in GGS (NC-3D: RSP) are depended with ordinary operator $\hat{x}_{i}$ and $p_{i}$ from the following projections relations:

$$
\begin{aligned}
& \delta \hat{x} \equiv \hat{x}-x=-\frac{\theta_{12}}{2} p_{y}-\frac{\theta_{13}}{2} p_{z} \\
& \delta \hat{y} \equiv \hat{y}-y=-\frac{\theta_{21}}{2} p_{x}-\frac{\theta_{23}}{2} p_{z} \\
& \delta \hat{z} \equiv \hat{z}-z=-\frac{\theta_{31}}{2} p_{x}-\frac{\theta_{32}}{2} p_{y}
\end{aligned}
$$

and

$$
\begin{aligned}
& \delta \hat{p}_{x} \equiv \hat{p}_{x}-p_{x}=\frac{\bar{\theta}_{12}}{2} y+\frac{\bar{\theta}_{13}}{2} z, \\
& \delta \hat{p}_{y} \equiv \hat{p}_{y}-p_{y}=\frac{\bar{\theta}_{21}}{2} x+\frac{\bar{\theta}_{23}}{2} z \\
& \delta \hat{p}_{z} \equiv \hat{p}_{z}-p_{z}=\frac{\bar{\theta}_{31}}{2} x+\frac{\bar{\theta}_{32}}{2} y
\end{aligned}
$$

Thus, the non-vanish 9-commutators in SP including in GGS (NC3D: RSP) can be determined as follows:

$$
\begin{aligned}
& {\left[\hat{x}, \hat{p}_{x}\right]=\left[\hat{y}, \hat{p}_{y}\right]=\left[\hat{z}, \hat{p}_{z}\right]=i,} \\
& {[\hat{x}, \hat{y}]=i \theta_{12},[\hat{x}, \hat{z}]=i \theta_{13},[\hat{y}, \hat{z}]=i \theta_{23}} \\
& {\left[\hat{p}_{x}, \hat{p}_{y}\right]=i \bar{\theta}_{12},\left[\hat{p}_{y}, \hat{p}_{z}\right]=i \bar{\theta}_{23} \text { and }\left[\hat{p}_{x}, \hat{p}_{z}\right]=i \bar{\theta}_{13}}
\end{aligned}
$$

And a similarly non-vanish 9-commutators in HP.The aim of this work is to study the MIHQP in NC 3-D space and phase to discover the new spectrum in this new symmetries from which other modified potentials are deduced as special cases, this potential plays an important role in many fields of physics such as molecular physics, solid state and chemical physics on based to the main reference [30,31] in QM and our previously works [10-29] in EQM. The rest of this search paper is

*Corresponding author: Abdelmadjid Maireche, Department of Physics, Laboratory of Physics and Material Chemistry, University of M'sila, M'sila 28000, Algeria, Tel: +213 664438317; E-mail: abmaireche@gmail.com

Received: October 30, 2017; Accepted: December 02, 2017; Published: December 09, 2017

Citation: Maireche A (2017) New Exact Non-relativistic Energy Eigen Values for Modified Inversely Quadratic Hellmann Plus Inversely Quadratic Potential. J Nanosci Curr Res 2:115. DOI: 10.4172/2572-0813.1000115

Copyright: @ 2017 Maireche A. This is an open-access article distributed under the terms of the Creative Commons Attribution License, which permits unrestricted use, distribution, and reproduction in any medium, provided the original author and source are credited. 
organized as follows: in the next section we briefly present and review the basic of eigenvalues and eignenfunctions for IHQP in ordinary 3-D spaces. In section 3, we give a brief review of Bopp's' shift method, then, we derive the spin-orbital NC Hamiltonians $\hat{H}_{s o-i h}$ for MIHQP in GGS (NC-3D: RSP), we find the exact spectrum produced by $\hat{H}_{s o-i h}$ by applying ordinary perturbation theory and then we deduce the exact spectrum produced by NC magnetic Hamiltonians $\hat{H}_{m-i h}$ for MIHQP in GGS (NC: 3D- RSP). In section four we resume the global spectrum for MIHQP and the main results. Finally, section 5 is kept for conclusive remarks.

\section{The IHQP in Ordinary 3-Dimensional Spaces}

The purpose of this section is to give a briefly review of eigenvalues and eignenfunctions for ordinary IHQP on based to the main reference [30],

$$
V(r)=b \delta^{2}-\frac{1}{r}(a+b \delta)+\frac{1}{r^{2}}(b+g)
$$

where $r$ represents the internuclear distance, $a$ and $\delta$ are the strengths of the coulomb and Yukawa potentials, respectively, while $\delta$ is the screening parameter. The ordinary SE with potential $V(r)$ can be written in spherical coordinate $(r, \theta, \varphi)$ as [30].

$$
\begin{aligned}
& \frac{1}{2 \mu}\left(-\frac{1}{r^{2}} \frac{\partial}{\partial r}\left(r^{2} \frac{\partial}{\partial r}\right)+\frac{1}{r^{2} \sin (\theta)} \frac{\partial}{\partial \theta}\left(\sin (\theta) \frac{\partial}{\partial \theta}\right)+\frac{1}{r^{2} \sin ^{2}(\theta)} \frac{\partial}{\partial \phi^{2}}\right) \Psi(r, \theta, \phi) \\
& +V(r) \Psi(r, \theta, \phi)=E \Psi(r, \theta, \phi)
\end{aligned}
$$

Where $E$ ordinary energies corresponding $V(r)$ in ordinary 3 -D spaces, the method of separation of variable has been applied in reference [30],

$$
\Psi(\vec{r})=\frac{R_{n l}(r)}{r} Y_{l m}(\theta, \phi)
$$

The radial function $R_{n l}(r)$ for IHQP satisfying the following differential equation [30],

$$
\frac{d^{2} R_{n l}(r)}{d r^{2}}+2 \mu\left(E-V(r)-\frac{\lambda}{2 \mu r^{2}}\right) R_{n l}(r)=0
$$

Here $\lambda=l(l+1)$, according NU method, the normalized energy eignenfunctions $\Psi(\vec{r})$ and corresponding eigenvalues $E$ for IHQP [30],

$$
\begin{aligned}
& \Psi(\ddot{r})=N_{n} z^{(-1+\sqrt{1+4 \gamma}) / 2} e^{-\sqrt{\alpha} z} L_{n}^{\sqrt{1+4 \gamma}}(2 \sqrt{\alpha} z) Y_{l m}(\theta, \phi) \\
& E=b \delta^{2}-\frac{\mu(a+b \delta)^{2} / 2}{\left(n+\frac{1}{2}+\sqrt{2 \mu(b+g)+(l+1 / 2)^{2}}\right)^{2}}
\end{aligned}
$$

Where $z=r^{2}, N_{n}$ is the normalization constant and the factor $\gamma=2 \mu(b+g)+l(l+1)$.

\section{NC 3-D Phase-Spaces GGS (NC-3D: RSP) Hamiltonians for MIHQP}

\section{Overview of the Formalism of Bopp's shift method}

We have been given a brief description of the MSE in GGS (NC3D: RSP) on based to our previously works [22-27]. To achieve this goal, we apply the important 4 -steps on the ordinary SE:

1-Ordinary 3-D Hamiltonian operator $\hat{H}_{i h}\left(p_{i}, x_{i}\right)$ will be replacing by new NC Hamiltonian operator $\hat{H}_{n c i h}\left(\hat{p}_{i}, \hat{x}_{i}\right)$.

2-Ordinary complex wave functions $\Psi(\vec{r})$ will be replacing by new complex wave function $\hat{\Psi}(\hat{\vec{r}})$.

3-Ordinary energies $E$ will be replaced by new values $E_{\text {ncih }}$.

While, the last step corresponds to replace the ordinary old product by new star product $(*)$, which allow us to constructing the MSE in GGS (NC-3D: RSP) as:

$$
\hat{H}_{n c i h}\left(\hat{p}_{i}, \hat{x}_{i}\right) * \widehat{\Psi}(\ddot{\vec{r}})=E_{n c i h} \widehat{\Psi}(\ddot{\vec{r}})
$$

The Bopp's shift method allows finding the reduced above MSE without star product as:

$$
H_{i h}\left(\hat{p}_{i}, \hat{x}_{i}\right) \Psi(\vec{r})=E_{n c i h} \Psi(\vec{r})
$$

the modified Hamiltonian operator $H_{i h}\left(\hat{p}_{i}, \hat{x}_{i}\right)$ for MIHQP obtained by replace both $x_{i}(x, y, z)$ and $p_{i}\left(p_{x}, p_{y}, p_{z}\right)$ by new two operators $\hat{x}_{i}$ and $\hat{p}_{i}$, respectively, in usual quantum Hamiltonian operator $H_{i h}\left(p_{i}, p_{i}\right)$ :

$$
\begin{aligned}
& V(r) \rightarrow V_{i h}(\hat{r})=\frac{b+g}{\hat{r}^{2}}-\frac{a+b \delta}{\hat{r}}+b \delta^{2} \\
& \frac{p_{i}^{2}}{2 \mu} \rightarrow \frac{\hat{p}_{i}^{2}}{2 \mu}
\end{aligned}
$$

Thus, we have:

$$
H_{i h}\left(\hat{p}_{i}, \hat{x}_{i}\right)=\frac{\hat{p}_{i}^{2}}{2 \mu}+V(\hat{r})
$$

On based to our references [26-29], we can write the two operators $\hat{r}^{2}$ and $\hat{p}^{2}$ in GGS (NC-3D: RSP) as follows:

$$
\begin{aligned}
& \hat{r}^{2}=r^{2}-\overrightarrow{\mathbf{L}} \vec{\Theta} \\
& \frac{\hat{p}^{2}}{2 \mu}=\frac{p^{2}}{2 \mu}+\frac{\overrightarrow{\mathbf{L}} \overrightarrow{\vec{\theta}}}{2 \mu}
\end{aligned}
$$

Where the two couplings $\mathbf{L} \Theta \equiv L_{x} \Theta_{12}+L_{y} \Theta_{23}+L_{z} \Theta_{13}$ and $\overrightarrow{\mathbf{L}} \overrightarrow{\bar{\theta}} \equiv L_{x} \bar{\theta}_{12}+L_{y} \bar{\theta}_{23}+L_{z} \bar{\theta}_{13} \quad\left(\Theta \equiv \frac{\theta}{2}\right)$. Now, after straightforward calculations one can obtains the different terms for MIHQP in GGS (NC-3D: RSP) as follows:

$$
\frac{b+g}{\hat{r}^{2}}=\frac{b+g}{r^{2}}+\frac{b+g}{r^{4}} \overrightarrow{\mathbf{L}} \vec{\Theta} \text {-and }-\frac{a+b \delta}{\hat{r}}=\frac{a+b \delta}{r}+\frac{a+b \delta}{2 r^{3}} \overrightarrow{\mathbf{L}} \vec{\Theta}
$$

Which allow us to writing the modified potential $V_{i t}(\hat{r})$ in GGS (NC3D: RSP) as follows:

$$
V_{i h}(\hat{r})=\frac{b+g}{r^{2}}-\frac{1}{r}(a+b \delta)+b \delta^{2}+V_{p e r t-i h}(r, \Theta, \bar{\theta}) \ldots
$$

Where the additive operator $V_{\text {pert-ih }}(r, \Theta, \bar{\theta})$ is given by:

$$
V_{\text {pert-ih }}(r, \Theta, \bar{\theta})=\left[\frac{b+g}{r^{4}}-\frac{a+b \delta}{2 r^{3}}\right] \overrightarrow{\mathbf{L}} \vec{\Theta}+\frac{\overrightarrow{\mathbf{L}} \overrightarrow{\vec{\theta}}}{2 \mu} .
$$

We can observe that the above operator is proportional with two infinitesimal parameters $\bar{\theta}$ and $\bar{\theta}$, thus, we considering as a perturbative term.

The Spin-Orbital NC Hamiltonian Operator for MIHQP in GGS (NC-3D: RSP)

In order to discover the new contribution of perturbative term $V_{\text {pert-ih }}(r, \Theta, \bar{\theta})$ for MIHQP, we turn to the case of spin $1 / 2$ particles described by the MSE, we make the following two simultaneously transformations: 


$$
\overrightarrow{\mathbf{L}} \vec{\Theta} \rightarrow 2 \Theta \vec{S} \vec{L} \quad \text { and } \quad \overrightarrow{\mathbf{L}} \vec{\theta} \rightarrow 2 \vec{\theta} \vec{S} \vec{L}
$$

Then the perturbed operator $V_{\text {pert-ih }}(r, \Theta, \bar{\theta})$ become as:

$$
V_{\text {pert }-i h}(r, \Theta, \bar{\theta})=2\left[\frac{b+g}{r^{4}}-\frac{a+b \delta}{2 r^{3}}+\frac{1}{2 \mu}\right] \overrightarrow{\mathbf{L}} \overrightarrow{\mathbf{S}} \ldots \ldots .
$$

Here $\vec{S}$ denote to the spin of a fermionic particle (like electron in Hydrogen atom). Now, it is possible to replace the spin-orbital interaction $\overrightarrow{\mathbf{L}} \vec{S}$ by $G^{2}=\frac{1}{2}\left(\vec{J}^{2}-\vec{L}^{2}-\vec{S}^{2}\right)$ to obtain directly the corresponding eigenvalues, and then new physical $V_{\text {pert-ih }}(r, \Theta, \bar{\theta})$ can be expressed as:

$$
V_{p e r t-i h}(r, \Theta, \bar{\theta})=\left[\frac{b+g}{r^{4}}-\frac{a+b \delta}{2 r^{3}}+\frac{1}{2 \mu}\right]\left(\vec{J}^{2}-\vec{L}^{2}-\vec{S}^{2}\right) \cdots \cdots
$$

As it well known, the 4-operators $\left(\vec{J}^{2}, \vec{L}^{2}, \vec{S}^{2}\right.$ and $\left.J_{z}\right)$ formed a complete basis on QM, then the operator $\left(\vec{J}^{2}-\vec{L}^{2}-\vec{S}^{2}\right)$ will be gives 2-eigenvalues $\quad k_{ \pm} \equiv \frac{1}{2}\left\{\left(l \pm \frac{1}{2}\right)\left(l+\frac{1}{2} \pm 1\right)+l(l+1)-\frac{3}{4}\right\}, \quad$ corresponding $j=l \pm \frac{1}{2}$ respectively [23-29]. Then, one can form a diagonal matrix $\hat{H}_{\text {so-ih }}$ of $\operatorname{order}(3 \times 3)$, with non null elements: $\left[\left(\hat{H}_{s o-i h}\right)_{11},\left(\hat{H}_{s o-i h}\right)_{22},\left(\hat{H}_{s o-i h}\right)_{33}\right]$ for MIHQP in GGS (NC-3D: RSP):

$$
\begin{aligned}
& \left(\hat{H}_{s o-i n}\right)_{11}=k_{+} \alpha\left\{\Theta\left(\frac{(b+g)}{r^{4}}-\frac{(a+b \delta)}{2 r^{3}}\right)+\frac{\bar{\theta}}{2 \mu}\right\} \text { if } j=l+\frac{1}{2} \Rightarrow \text { spin up } \\
& \left(\hat{H}_{s o-i i}\right)_{22}=k_{-} \alpha\left\{\Theta\left(\frac{b+g}{r^{4}}-\frac{a+b \delta}{2 r^{3}}\right)+\frac{\bar{\theta}}{2 \mu}\right\} \mathrm{if} j=l-\frac{1}{2} \Rightarrow \text { spin down } \cdots \cdots \\
& \left(\hat{H}_{s o-i i h}\right)_{33}=0
\end{aligned}
$$

After profound calculation, one can show that, the radial function $R_{n l}(r)$ for MIHQP satisfying the following differential equation, in EQM structure of GGS (NC-3D: RSP):

$$
\frac{d^{2} R_{n l}(r)}{d r^{2}}+2 \mu\left(E-V_{\text {ih }}(r)-\frac{\lambda}{2 \mu r^{2}}-2\left[\frac{b+g}{r^{4}}-\frac{a+b \delta}{2 r^{3}}+\frac{1}{2 \mu}\right] \overrightarrow{\mathbf{L}} \overrightarrow{\mathbf{S}}\right) R_{n l}(r)=0
$$

The Exact Spectrum Produced by NC Spin-Orbital Hamiltonian $\hat{H}_{s o-i h}$ for MIHQP using Standard Perturbation Theory in GGS (NC- 3D: RSP)

The aim of this subsection is to obtain the modifications to the energy levels for $n^{\text {th }}$ excited states $E_{\text {u-ih }}$ and $E_{\text {d-ih }}$ corresponding a fermionic particle with two polarizations spin up and spin down, respectively, at first order of two infinitesimal parameters $\Theta$ and $\bar{\theta}$. In order to achieve this goal, we apply the standard perturbation theory using eq. (14) for MIHQP:

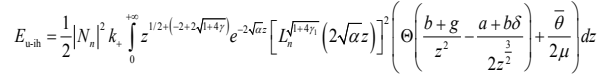

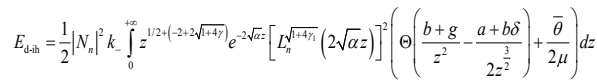

It is possible to write both $E_{\text {u-ih }}$ and $E_{\mathrm{d} \text {-ih }}$ as functions of three factors $T_{i h}^{1}, T_{i h}^{2}$ and $\bar{T}_{i h}$ as follow:

$$
\begin{aligned}
& E_{\mathrm{u}-\mathrm{ih}}=\frac{1}{2}\left|N_{n}\right|^{2} k_{+}\left\{\Theta\left(T_{i h}^{1}+T_{i h}^{2}\right)+\frac{\bar{\theta}}{2 \mu} \bar{T}_{i h}\right\} \\
& E_{\mathrm{d}-\mathrm{h}}=\frac{1}{2}\left|N_{n}\right|^{2} k_{-}\left\{\Theta\left(T_{i h}^{1}+T_{i h}^{2}\right)+\frac{\bar{\theta}}{2 \mu} \bar{T}_{i h}\right\}
\end{aligned}
$$

The explicit mathematical forms of 3- factors $T_{i h}^{1}, T_{i h}^{2}$ and $\bar{T}_{i h}$ are given by:

$$
\begin{aligned}
& T_{i h}^{1}=(b+g) \int_{0}^{+\infty} z^{\sqrt{4+16 \gamma}-5 / 2-1} e^{-2 \sqrt{\alpha z}}\left[L_{n}^{\sqrt{1+4 y}}(2 \sqrt{\alpha} z)\right]^{2} d z, \\
& T_{y h}^{2}=-\frac{(a+b \delta)^{+\infty}}{2} \int_{0}^{+\infty} z^{(2-\sqrt{4+16 \gamma})-1} e^{-2 \sqrt{\alpha z}}\left[L_{n}^{\sqrt{1+4 y}}(2 \sqrt{\alpha} z)\right]^{2} d z, \ldots \ldots . . \\
& \bar{T}_{i h}=\int_{0}^{+\infty} z^{(\sqrt{1+4 y}-1 / 2)-1} e^{-2 \sqrt{\alpha z}}\left[L_{n}^{\sqrt{1+4 \gamma}}(2 \sqrt{\alpha} z)\right]^{2} d z
\end{aligned}
$$

Applying the following special integration [32],

$$
\begin{aligned}
& \int_{0}^{+\infty} t^{\alpha-1} \exp (-\delta t) L_{m}^{\lambda}(\delta t) L_{n}^{\beta}(\delta t) d t=\frac{\delta^{-\alpha} \Gamma(n-\alpha+\beta+1) \Gamma(m+\lambda+1)}{m ! n ! \Gamma(1-\alpha+\beta) \Gamma(1+\lambda)} \ldots . \\
& { }_{3} F_{2}(-m, \alpha, \alpha-\beta ;-n+\alpha, \lambda+1 ; 1)
\end{aligned}
$$

To obtain the modifications to the energy levels for $n^{\text {th }}$ excited states, where ${ }_{3} F_{2}(-m, \alpha, \alpha-\beta ;-n+\alpha, \lambda+1 ; 1)$ denote to the hypergeometric function, obtained from ${ }_{p} F_{q}\left(\alpha_{1}, \ldots, \alpha_{p}, \beta_{1}, \ldots, \beta_{q}, z\right)$ for $p=3$ and $q=2$. After straightforward calculations, we can obtain the explicitly results:

$$
\begin{aligned}
& T_{i h}^{1}=(b+g) \frac{(8 \sqrt{1+4 \gamma}-5)^{(5 / 4-\sqrt{1+4 \gamma})} \Gamma(n-\sqrt{1+4 \gamma}+7 / 21) \Gamma(n+\sqrt{1+4 \gamma}+1)}{n ! \Gamma(7 / 2-\sqrt{1+4 \gamma}) \Gamma(1+\sqrt{1+4 \gamma})} \\
& { }_{3} F_{2}(-n, 2 \sqrt{1+4 \gamma}-5 / 2, \sqrt{1+4 \gamma}-5 / 2 ;-n+2 \sqrt{1+4 \gamma}-5 / 2, \sqrt{1+4 \gamma}+1 ; 1) \\
& T_{i h}^{2}=-\frac{(a+b \delta)}{2} \frac{(8-\sqrt{64+256 \gamma})^{(\sqrt{114+\gamma}-1 / 2)} \Gamma(n+3 \sqrt{1+4 \gamma}-1) \Gamma(n+\sqrt{1+4 \gamma}+1)}{n ! \Gamma(3 \sqrt{1+4 \gamma}-1) \Gamma(1+\sqrt{1+4 \gamma})} \\
& { }_{3} F_{2}(-n, 2-\sqrt{4+16 \gamma}, 2-3 \sqrt{1+4 \gamma} ;-n+2-\sqrt{4+16 \gamma}, \sqrt{1+4 \gamma}+1 ; 1) \\
& \bar{T}_{i n}=\frac{(\sqrt{16+64 \gamma}-2)^{1 / 4-\sqrt{1 / 4+\gamma}} \Gamma(n+3 / 2) \Gamma(n+\sqrt{1+4 \gamma}+1)}{n !^{2} \Gamma(3 / 2) \Gamma(1+\sqrt{1+4 \gamma})} \\
& { }_{3} F_{2}(-n, \sqrt{1+4 \gamma}-1 / 2,-1 / 2 ;-n+\sqrt{1+4 \gamma}-1 / 2, \sqrt{1+4 \gamma}+1 ; 1)
\end{aligned}
$$

Inserting the above obtained expressions into equations (28), gives the following results for exact modifications $E_{\text {u-ih }}$ and $E_{\text {d-ih }}$ produced by new spin-orbital operator effect for MIHQP:

$$
\begin{aligned}
& E_{\mathrm{u}-\mathrm{ih}}=\frac{1}{2}\left|N_{n}\right|^{2} k_{+}\left\{\Theta T_{i h}+\frac{\bar{\theta}}{2 \mu} \bar{T}_{i h}\right\} \\
& E_{\mathrm{d}-\mathrm{ih}}=\frac{1}{2}\left|N_{n}\right|^{2} k_{-}\left\{\Theta T_{i h}+\frac{\bar{\theta}}{2 \mu} \bar{T}_{i h}\right\}
\end{aligned}
$$

Where the new factor $T_{i h}$ is sum of two factors $T_{i h}^{1}$ and $T_{i h}^{2}$.

\section{The Exact Spectrum Produced by NC Magnetic Hamiltonians $\hat{H}_{m-i h}$ for MIHQP in GGS (NC-3D: RSP)}

Having found out how to calculate the corrections of energies for the automatically produced spin-orbital, we can discover a second symmetry produced by the effect and influence of the noncommutativity of space-phase, known by modified Zeeman Effect for MIHQP, to found this physical symmetry we apply the same strategy in our previously works as follows [25-29],

$\Theta \rightarrow \chi B \quad$ and $\quad \bar{\theta} \rightarrow \bar{\sigma} B$

The two parameters $\chi$ and $\bar{\sigma}$ are just only infinitesimal real 
proportional's constants and $B$ is a uniform external magnetic field, we orient it to $(o z)$ axis and then we can make the following two translations for MIHQP:

$$
\left\{\Theta\left(\frac{b+g}{r^{4}}-\frac{a+b \delta}{2 r^{3}}\right)+\frac{\bar{\theta}}{2 \mu}\right\} \vec{B} \vec{L} \rightarrow B\left(\chi\left(\frac{b+g}{r^{4}}-\frac{a+b \delta}{2 r^{3}}\right)+\frac{\bar{\sigma}}{2 \mu}\right) L_{z}
$$

Which allow us to introduce the modified new magnetic Hamiltonians $\hat{H}_{m-i h}$ in GGS (NC-3D: RSP) for MIHQP, as:

$$
\hat{H}_{m-i h}=\left(\chi\left(\frac{b+g}{r^{4}}-\frac{a+b \delta}{2 r^{3}}\right)+\frac{\bar{\sigma}}{2 \mu}\right)\left(\vec{B} \vec{J}+\hat{H}_{z}\right) \ldots \ldots
$$

Where $\hat{H}_{z} \equiv-\vec{S} \vec{B}$ denote to the ordinary operator of Hamiltonian for Zeeman Effect in QM. To obtain the exact NC magnetic modifications of energy $E_{\text {mag-ih }}$ for MIHQP, it is sufficient to replace the 3-parameters $k_{+}, \Theta$ and $\bar{\theta}$ in the eq. (34) by the following new 3-parameters $m, \chi$ and $\bar{\sigma}$ :

$$
E_{\text {mag-ih }}=\frac{1}{2}\left|N_{n}\right|^{2} B\left\{\chi T_{i h}+\frac{\bar{\sigma}}{2 \mu} \bar{T}_{i h}\right\} m \text {. }
$$

Where $m$ denote to the eigenvalues of the operator $L_{z}$ which can be taking the discrete atomic values $-l,-l+1, \ldots, 0, \ldots, l$.

\section{Results}

Let us now resume the global exact spectrum of $n^{\text {th }}$ excited states ( $E_{\text {ncu-ih }}, E_{\text {ncd-ih }}$ and $E_{\text {com-ih }}$ ) for MIHQP in GGS (NC-3D: RSP) produced by the diagonal elements $\left[\left(\hat{H}_{n c-i h}\right)_{11},\left(\hat{H}_{n c-i h}\right)_{22}\right.$ and $\left(\hat{H}_{n c-i h}\right)_{33}$ ] of the operator $\hat{H}_{n c-i h}$ :

$$
\begin{aligned}
& \left(\hat{H}_{n c-i h}\right)_{11}=-\frac{\Delta}{2 \mu}+\frac{b+g}{r^{2}}-\frac{a+b \delta}{r}+b \delta^{2}+k_{+}\left\{\Theta\left(\frac{b+g}{r^{4}}-\frac{a+b \delta}{2 r^{3}}\right)+\frac{\bar{\theta}}{2 \mu}\right\} \\
& +\left(\chi\left(\frac{\left(b-V_{0}\right)}{r^{4}}+\frac{\left(2 V_{0}-a-b \delta\right)}{2 r^{3}}\right)+\frac{\bar{\sigma}}{2 \mu}\right) B L_{z} \quad \text { if } j=l+\frac{1}{2} \Rightarrow \text { spin -up } \\
& \left(\hat{H}_{n c-i h}\right)_{22}=-\frac{\Delta}{2 \mu}+\frac{b+g}{r^{2}}-\frac{a+b \delta}{r}+b \delta^{2}+k_{-}\left\{\Theta\left(\frac{b+g}{r^{4}}-\frac{a+b \delta}{2 r^{3}}\right)+\frac{\bar{\theta}}{2 \mu}\right\} \\
& +\left(\Theta\left(\frac{\left(b-V_{0}\right)}{r^{4}}-\frac{a+b \delta}{2 r^{3}}\right)+\frac{\bar{\theta}}{2 \mu}\right) B L_{z} \quad \text { if } j=l-\frac{1}{2} \Rightarrow \text { spin -down } \\
& \left(\hat{H}_{n c-i h}\right)_{33}=-\frac{\Delta}{2 \mu}+\frac{b+g}{r^{2}}-\frac{a+b \delta}{r}+b \delta^{2} \ldots \ldots \ldots \ldots \ldots . . . .
\end{aligned}
$$

As follows:

$$
\begin{aligned}
& E_{\text {ncuih }}=b \delta^{2}-\frac{\mu(a+b \delta)^{2} / 2}{\left(n+\frac{1}{2}+\sqrt{2 \mu(b+g)+(l+1 / 2)^{2}}\right)^{2}}+\ldots \ldots . . \\
& +\frac{\left|N_{n}\right|^{2}}{2} k_{+}\left\{\Theta T_{i h}+\frac{\bar{\theta}}{2 \mu} \bar{T}_{i h}\right\}+\frac{\left|N_{n}\right|^{2}}{2} B\left\{\chi T_{i h}+\frac{\bar{\sigma}}{2 \mu} \bar{T}_{i h}\right\} m \\
& E_{\text {ncdih }}=b \delta^{2}-\frac{\mu(a+b \delta)^{2} / 2}{\left(n+\frac{1}{2}+\sqrt{2 \mu(b+g)+(l+1 / 2)^{2}}\right)^{2}}+ \\
& +\frac{\left|N_{n}\right|^{2}}{2} k_{-}\left\{\Theta T_{i h}+\frac{\bar{\theta}}{2 \mu} \bar{T}_{i h}\right\}+\frac{\left|N_{n}\right|^{2}}{2} B\left\{\chi T_{i h}+\frac{\bar{\sigma}}{2 \mu} \bar{T}_{i h}\right\} m \\
& E_{\text {com-ih }}=b \delta^{2}-\frac{\mu(a+b \delta)^{2} / 2}{\left(n+\frac{1}{2}+\sqrt{2 \mu(b+g)+(l+1 / 2)^{2}}\right)^{2}} . .
\end{aligned}
$$

It is well known that the atomic quantum number $m$ can be takes $(2 l+1)$ values and we have also two possible values for eigenvalues $j=l \pm \frac{1}{2}$, thus every state in usually 3 -dimensional space for MIHQP will be replace, in GGS (NC-3D: RSP) by $2(2 l+1)$ sub-states and then the degenerated state can be take $2 \sum_{i=0}^{n-1}(2 l+1) \equiv 2 n^{2}$ values. It is important to noticing that our recent study can be extended to apply to molecular $\left(\operatorname{spin} s \neq \frac{1}{2}\right)$, we replace the one of two factors $k_{ \pm} \equiv \frac{1}{2}\left\{\left(l \pm \frac{1}{2}\right)\left(l+\frac{1}{2} \pm 1\right)+l(l+1)-\frac{3}{4}\right\}$ by new factor $k(j, l, s) \equiv \frac{1}{2}\{j(j+1)+l(l+1)-s(s+1)\}$ with $|l-s| \leq j \leq|l+s|$, which allow us to obtaining the new modifications to the energy levels $E_{\text {nc-ih }}$ for MIHQP:

$$
\begin{aligned}
& E_{\text {ncih }}=b \delta^{2}-\frac{\mu(a+b \delta)^{2} / 2}{\left(n+\frac{1}{2}+\sqrt{2 \mu(b+g)+(l+1 / 2)^{2}}\right)^{2}}+ \\
& +\frac{\left|N_{n}\right|^{2}}{2} k(j, l, s)\left\{\Theta T_{i h}+\frac{\bar{\theta}}{2 \mu} \bar{T}_{i h}\right\}+\frac{\left|N_{n}\right|^{2}}{2} B\left\{\chi T_{i h}+\frac{\bar{\sigma}}{2 \mu} \bar{T}_{i h}\right\} m
\end{aligned}
$$

And the corresponding NC Hamiltonian operators $\hat{H}_{n c-i h}$ can be fixed by the following results:

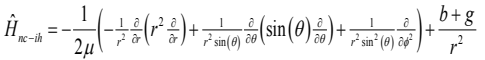

$$
\begin{aligned}
& -\frac{a+b \delta}{r}+b \delta^{2}+\left\{\Theta\left(\frac{(b+g)}{r^{4}}-\frac{a+b \delta}{2 r^{3}}\right)+\frac{\bar{\theta}}{\mu}\right) \overrightarrow{\mathbf{L}} \overrightarrow{\mathbf{S}}+\left(\chi\left(\frac{(b+g)}{r^{4}}-\frac{a+b \delta}{2 r^{3}}\right)+\frac{\bar{\sigma}}{2 \mu}\right) B L_{z}
\end{aligned}
$$

We now look at some special cases and relationships between our recently results and some other existing results in our previously works.

Case 1: If we set the parameters, $(b=g=0)$ and $a=z e^{2}$ it is easy to show that equations (21) and (34) are reduce to the modified interaction $V_{\text {pert-col }}(r, \Theta, \bar{\theta})$ of a particle in the modified Coulomb potential and corresponding NC spectrum $E_{\text {nc-ih }}$, respectively.

$$
\begin{aligned}
& V_{\text {pert-ih }}(r, \Theta, \bar{\theta}) \rightarrow V_{\text {pert-col }}(r, \Theta, \bar{\theta}) \equiv-\frac{z e^{2}}{2 r^{3}} \overrightarrow{\mathbf{L}} \vec{\Theta}+\frac{\overrightarrow{\mathbf{L}} \overrightarrow{\bar{\theta}}}{2 \mu} . \cdot \\
& E_{\text {ncin }} \rightarrow E_{\text {ncilh }} \equiv-\frac{Z^{2} \mu e^{4}}{2(n+l+1)}+\frac{\left|N_{n}\right|^{2}}{2} k(j, l, s)\left\{\Theta T_{i h}+\frac{\bar{\theta}}{2 \mu} \bar{T}_{i h h}\right\}(b=g=0) \\
& +\frac{\left|N_{n}\right|^{2}}{2} B\left\{\chi T_{i h}+\frac{\bar{\sigma}}{2 \mu} \bar{T}_{i h}\right\}(b=g=0) m
\end{aligned}
$$

With $\gamma=l(l+1)$ and the three factors $T_{i t}^{1}(b=g=0), \bar{T}_{i t}(b=g=0)$ and $T_{i t}^{2}(b=g=0)$ are given explicitly by:

$$
\begin{aligned}
& T_{i h}^{1}(b=g=0)=0 \\
& T_{i h}^{2}(b=g=0)=-\frac{a}{2} \frac{(8-\sqrt{64+256 \gamma})^{(\sqrt{114 \gamma-11 / 2})} \Gamma(n+3 \sqrt{1+4 \gamma}-1) \Gamma(n+\sqrt{1+4 \gamma}+1)}{n ! \Gamma(3 \sqrt{1+4 \gamma}-1) \Gamma(1+\sqrt{1+4 \gamma})} \\
& { }_{3} F_{2}(-n, 2-\sqrt{4+16 \gamma}, 2-3 \sqrt{1+4 \gamma} ;-n+2-\sqrt{4+16 \gamma}, \sqrt{1+4 \gamma}+1 ; 1) \\
& \bar{T}_{\mu}(b=g=0)=\bar{T}_{\mu}
\end{aligned}
$$

Case 2: Similarly, if we set $g=0$ and $(a, b) \neq(0,0)$ equations (21) and (34) are reduce to the results of modified inversely quadratic Hellmann potential in $V_{\text {pert- } h}(r, \Theta, \bar{\theta})$ and corresponding modified bound state energy spectrum $E_{\mathrm{nch}}$ of a vibrating rotating diatomic molecule, respectively.

$$
\begin{aligned}
& V_{\text {pert-in }}(r, \Theta, \bar{\theta}) \rightarrow V_{\text {pert }-\mathrm{h}}(r, \Theta, \bar{\theta}) \equiv\left[\frac{b}{r^{4}}-\frac{a+b \delta}{2 r^{3}}\right] \overrightarrow{\mathbf{L}} \vec{\Theta}+\frac{\overrightarrow{\mathbf{L}} \bar{\theta}}{2 \mu} \\
& E_{\text {ncin }} \rightarrow E_{\text {nch }} \equiv b \delta^{2}-\frac{\mu(a+b \delta)^{2} / 2}{\left(n+\frac{1}{2}+\sqrt{2 \mu b+(l+1 / 2)^{2}}\right)^{2}}+ \\
& \frac{\left|N_{n}\right|^{2}}{2} k(j, l, s)\left\{\Theta T_{i n}+\frac{\bar{\theta}}{2 \mu} \bar{T}_{i n}\right\}(g=0)+\frac{\left|N_{n}\right|^{2}}{2} B\left\{\chi T_{i n}+\frac{\bar{\sigma}}{2 \mu} \bar{T}_{i n}\right\}(g=0) m
\end{aligned}
$$


With $\gamma=2 \mu b+l(l+1)$ and the three factors $T_{i h}^{1}(g=0), \bar{T}_{u l}(g=0)$ and $T_{i h}^{2}(g=0)$ are given explicitly by:

$$
\begin{aligned}
& T_{i h}^{1}(g=0)=b \frac{(8 \sqrt{1+4 \gamma}-5)^{(5 / 4-\sqrt{1+4 \gamma})} \Gamma(n-\sqrt{1+4 \gamma}+7 / 21) \Gamma(n+\sqrt{1+4 \gamma}+1)}{n !^{2} \Gamma(7 / 2-\sqrt{1+4 \gamma}) \Gamma(1+\sqrt{1+4 \gamma})} \\
& { }_{3} F_{2}(-n, 2 \sqrt{1+4 \gamma}-5 / 2, \sqrt{1+4 \gamma}-5 / 2 ;-n+2 \sqrt{1+4 \gamma}-5 / 2, \sqrt{1+4 \gamma}+1 ; 1) \\
& T_{i h}^{2}(g=0)=T_{i h}^{2} \quad \text { and } \bar{T}_{i j}(g=0)=\bar{T}_{\text {ih }}
\end{aligned}
$$

It is important to notice that, the appearance of the polarization states of a fermionic particle for MIHQP in the non-relativistic MSE indicates a validity of obtained results at high energy where the two relativistic equations Klein-Gordon and Dirac are applied; this gives a positive indication of the possibility to apply these results of various Nano-particles at nano scales. Finally, if we make the two simultaneously limits $(\theta, \bar{\theta}) \rightarrow(0,0)$ we obtain all results of QM which indicates the validity of our research.

\section{Conclusion}

In this article, the Bopp's' shift method has been studied and applied to MIHQP and the corresponding new energy eigenvalues of MSE are successfully investigated by applying the standard perturbation theory in GGS (NC-3D: RSP), we showed the obtained degenerated spectrum depended by ordinary discrete atomic quantum numbers $\left(m, j=l \pm \frac{1}{2}\right.$ and $S_{z}= \pm \frac{1}{2}$ ). Furthermore, the validity of obtained corrections can be prolonged to Nano-particles at Nano and Plank's scales. In addition, we recover the ordinary commutative spectrums when, we make the two simultaneously limits: $(\theta, \bar{\theta}) \rightarrow(0,0)$ for MIHQP in GGS (NC-3D: RSP). The results are in excellent agreement with our reference no. [16].

\section{Acknowledgments}

The research leading to this article was supported with search Laboratory of Physics and Material Chemistry, Physics department, Sciences Faculty, University of M'sila, Algeria. The author is grateful to the reviewers for comments, which have significantly help for the improvement of this present search work.

\section{References}

1. Connes A, Douglas MR, Schwarz A (1998) Noncommutative geometry and matrix theory: compactification on tori. JHEP 9802: 003.

2. Djemei AEF, Smail H (2004) On quantum mechanics on noncommutative quantum phase space. Commun Theor Phys 41: 837-844.

3. Lee J (2005) Star products and the landau problem. J Korean Phys Soc 47: 571-576.

4. Yuan $Y(2010)$ Spin $1 / 2$ relativistic particle in a magnetic field in NC Ph. Chin Phys C 34:543.

5. Hoseini F, Saha JK, Hassanabadi H (2016) Investigation of fermions in noncommutative space by considering kratzer potential. Commun Theor Phys 65: 695-700.

6. Bertolami O, Leal P (2015) Aspects of phase-space noncommutative quantum mechanics. Phys Lett B 750: 6-11.

7. Bastos C, Bertolami O, Dias NC, Prata JN (2008) Weyl-Wigner formulation of noncommutative quantum mechanics. J Math Phys 49: 072101.

8. Gamboa J, Loewe M, Rojas JC (2001) Noncommutative quantum mechanics. Phys Rev D 64: 06790.

9. Chaichian M, Sheikh-Jabbari MM, Tureanu A (2001) Hydrogen atom spec-trum and the Lamb shift in noncommutative QED. Phys Rev Lett 86: 2716.

10. Maireche A (2015) Nonrelativistic atomic spectrum for companied harmonic oscillator potential and its inverse in both nc-2d: RSP. ILCPA 56: 1-9.

11. Maireche A (2015) A new approach to the non relativistic schrödinge equation for an energy-depended potential $\mathrm{v}(\mathrm{r}, \mathrm{en}, \mathrm{I})=\mathrm{v} 0(1+n e n, \mathrm{I}) \mathrm{r} 2$ in both noncommutative three dimensional spaces and phases. ILCPA 60: 11-19.
12. Maireche A (2017) Effects of two-dimensional noncommutative theories on bound states schrodinger diatomic molecules under new modified kratzer-type interactions. ILCPA 76: 1-11.

13. Maireche $A$ (2015) Spectrum of hydrogen atom ground state counting quadratic term in schrödinger equation. Afr Rev Phys 10: 177-183.

14. Maireche A (2016) A new nonrelativistic investigation for the lowest excitations states of interactions in one-electron atoms, muonic, hadronic and rydberg atoms with modified inverse power potential. International Frontier Science Letters 9: 33-46.

15. Maireche A (2017) New bound states for modified vibrational-rotational structure of supersingular plus coulomb potential of the schrödinger equation in one-electron atoms. Int Lett Chem Phys Astron 73: 31-45.

16. Abdelmadjid Maireche (2016) New exact energy eigen-values for (MIQYH) and (MIQHM) central potentials: Non-relativistic solutions. Afr Rev Phys 11: 175-184.

17. Maireche A (2015) Deformed quantum energy spectra with mixed harmonic potential for nonrelativistic schrödinger equation. J Nano Electron Phys 7: 6 .

18. Maireche A (2015) A recent study of quantum atomic spectrum of the lowest excitations for schrödinger equation with typical rational spherical potential at planck's and nanoscales. J Nano Electron Phys 7: 7.

19. Maireche A (2016) The non-relativistic ground state energy spectra of potential counting coulomb and quad-ratic terms in non-commutative two dimensional real spaces and phases. J Nano Electron Phys 8: 6.

20. Maireche A (2016) A new theoretical study of quantum atomic energy spectra for lowest excited states of central (pihoiq) potential in noncommutative spaces and phases symmetries at plank's and nanoscales. J Nano Electron Phys 8: 10.

21. Maireche A (2016) A new nonrelativistic atomic energy spectrum of energy dependent potential for heavy quarkouniom in noncommutative spaces and phases symmetries. J Nano Electron Phys 8: 6.

22. Maireche A (2015) New exact solution of the bound states for the potentia family $v(r)=a / r 2-b / r+c r k(k=0,-1,-2)$ in both noncommutative three dimensional spaces and phases: non relativistic quantum mechanics. Int Lett Chem Phys Astron 58: 164-176

23. Maireche A (2015) Quantum hamiltonian and spectrum of schrödinger equation with companied harmonic oscillator potential and its inverse in both three dimensional non-commutative real space and phase. J Nano Electron Phys 7: 7.

24. Maireche A (2015) A new study to the schrödinger equation for modified potential $V(r)=a r 2+b r-4+c r-6$ in non-relativistic three dimensional real spaces and phases. ILCPA 61: 38-48.

25. Maireche A (2016) A new nonrelativistic investigation for spectra of heav quarkonia with modified cornell potential: noncommutative three dimensional space and phase space solutions. J Nano Electron Phys 8: 9.

26. Maireche A (2015) New exact bound states solutions for (C.F.P.S.) potentia in the case of Non-commutative three dimensional non relativistic quantum mechanics. Med J Model Simul 4: 060-072.

27. Maireche A (2016) New bound state energies for spherical quantum dots in presence of a confining potential model at nano and plank's scales. NanoWorld J 1: 122-129.

28. Abdelmadjid Maireche (2015) New quantum atomic spectrum of Schrödinge equation with pseudo harmonic potential in both non-commutative three dimensional spaces and phases. Lat Am J Phys Educ 9: 1301.

29. Abdelmadjid Maireche (2016) A complete analytical solution of the mie-type potentials in non-commutative 3-dimensional spaces and phases symmetries. Afr Rev Phys 11: 111-117.

30. Ita BI, Ehi-Eromosele CO, Edobor-Osoh A, Ikeuba AI (2014) Solutions of the Schrödinger equation with inversely quadratic hellmann plus inversely quadratic potential using nikiforov-uvarov method. application of mathematics in technical and natural sciences. AIP Conf Proc 1629: 360-362.

31. Hitler L, Ita BI, Isa PA, Nelson NI, Joseph I, et al. (2017) Wkb solutions for inversely quadratic yukawa plus inversely quadratic hellmann potential. World Journal of Applied Physics 2:109-112.

32. Abramowitz M, Stegun IA (1965) Handbook of mathematical functions with formulas, graphs and mathematical tables. Dover Publications, New York. 